\title{
Transformación de requisitos representados en esquemas preconceptuales a modelos de interacción de sistemas holónicos
}

\author{
Transforming requirements represented by pre-conceptual \\ schemas into interaction models of holonic systems \\ Carlos M. Zapata ${ }^{1} \quad$ Gloria L. Giraldo ${ }^{1} \quad$ Germán Zapata $^{2} \quad$ Adrián S. Arboleda ${ }^{3}$ \\ Recibido 8 de noviembre de 2010, aceptado 16 de diciembre de 2013 \\ Received: November 8, 2010 Accepted: December 16, 2013
}

\begin{abstract}
RESUMEN
Los sistemas holónicos de manufactura buscan mejorar la productividad de las organizaciones y dilucidar sus requisitos por medio de la planificación de estrategias para alcanzar sus objetivos de producción. Algunos diseños arquitectónicos se proponen para determinar el comportamiento y estructura de un holón. Diferentes autores analizan el desarrollo de estos sistemas y su posible implantación en las organizaciones, como lo plantea la metodología ANEMONA. Sin embargo, la educción de requisitos para sistemas holónicos es un proceso largo, con debilidades en la representación e identificación de holones. Por su parte, los esquemas preconceptuales (EP) son herramientas útiles para la educción de requisitos, pues son fácilmente comprensibles y tratables computacionalmente. En este artículo se propone la representación de requisitos para sistemas holónicos mediante EP y la formulación de reglas de conversión para la generación de modelos de interacción basados en ANEMONA desde EP. Este enfoque mejora el análisis de sistemas holónicos en un dominio particular porque se obtiene una identificación rápida de posibles holones, se logra entender fácilmente las interacciones de todos los actores en un proceso específico y se elimina la ambigüedad en una descripción de procesos mediante el uso de EP. Un caso de estudio demuestra las ventajas de la representación en EP y de la aplicación de las reglas de transformación.
\end{abstract}

Palabras clave: Sistemas holónicos, ingeniería de requisitos, modelado, esquemas preconceptuales, modelos de interacción.

\begin{abstract}
Holonic manufacturing systems search for improvement of the organizational productivity and elucidation of the requirements by means of strategy planning in order to reach production goals. Some architectural designs have been proposed to determine the behavior and structure of a holon. Various authors examine the development of these systems and the appropriate implementation in organizations, as suggested by the ANEMONA methodology. However, the requirements elicitation for holonic systems is a long process, whose drawbacks are found on representing and identifying holons. Pre-conceptual schemas (PS) are useful requirement elicitation tools, because they have computational tractability and they can be easily understood. In this paper we propose the representation of requirements for holons systems in PS and the formulation of conversion rules for generating the ANEMONA-based interaction model from EP. This approach is intended to improve the analysis of holonic systems in a particular domain, providing
\end{abstract}

\footnotetext{
1 Departamento de Ciencias de la Computación y de la Decisión. Facultad de Minas. Universidad Nacional. Sede Medellín. Medellín, Colombia. E-mail: cmzapata@bt.unal.edu.co; glgiraldog@unal.edu.co

2 Departamento de Energía Eléctrica y Automática. Facultad de Minas. Universidad Nacional. Sede Medellín. Medellín, Colombia. E-mail: gdzapata@unal.edu.co

3 Grupo T\&T. Facultad de Minas. Universidad Nacional. Sede Medellín. Medellín, Colombia. E-mail: asarbolec@unal.edu.co
} 
a fast identification of possible holons and making the interactions among actors easily understood in a specific process and ambiguity is suppressed from the process description by using PS. The advantages of the PS-based representation and the use of transformation rules by using a case study are shown.

Keywords: Holonic systems, requirements engineering, modeling, pre-conceptual schemas, interaction models.

\section{INTRODUCCIÓN}

Hacia finales de la década de los 80 del siglo pasado comenzó a surgir un interés, tanto desde la parte académica como desde el área industrial, por la necesidad de mejorar la productividad en la empresa manufacturera. Las empresas requerían alternativas conceptuales para alcanzar características como agilidad, flexibilidad y administración inteligente. Fue a principios de los 90 de ese mismo siglo que los sistemas holónicos de manufactura (HMS) se concibieron, trayendo consigo una nueva visión en las fábricas manufactureras [1].

Con el nuevo paradigma surgió la necesidad de plantear arquitecturas que modelaran el pensamiento holónico. La arquitectura más representativa fue PROSA, que dio el soporte a las primeras implementaciones de fábrica. PROSA se destaca por el intercambio de información entre los tres holones básicos que simulan el conocimiento del proceso, del producto y de la ejecución [2]. A partir de PROSA se desprenden otras arquitecturas como ADACOR y HDC, las cuales añaden más inteligencia a los holones básicos, integrados con la supervisión de sus operaciones [3-4].

Luego de unos años, las empresas empezaron a exigir implementaciones holónicas en las fábricas. El desarrollo de software hacia el paradigma comenzó con metodologías orientadas a objetos [5], para luego evolucionar a metodologías multiagente, debido a sus semejanzas con los holones [6]. Giret [7] construye una metodología para desarrollo de sistemas holónicos llamada ANEMONA, basada en sistemas multiagente, con una fase de requisitos claramente que delimita varios modelos de análisis, entre ellos el de interacción. Sin embargo, las adaptaciones desde agentes hacia sistemas holónicos carecen de una representación de requisitos sencilla, válida y computacionalmente tratable para fases de diseño e implementación.
En el campo de la ingeniería de requisitos, los esquemas preconceptuales (EP) surgen como un instrumento de ayuda para la representación de la terminología de un dominio y facilitar su entendimiento, tanto para analistas como para interesados [8]. Su aporte radica en la construcción sencilla de nociones básicas alrededor de una problemática para una posterior traducción a esquemas conceptuales, tales como diagramas UML.

En este artículo se propone una representación de requisitos de sistemas holónicos por medio de esquemas preconceptuales, para facilitar a los analistas de software la identificación de holones y la generación del diagrama de interacción propuesto en ANEMONA.

La estructura de este artículo es la siguiente: primero se introducen los conceptos de los sistemas holónicos. A continuación se exponen arquitecturas holónicas representativas. En la siguiente sección se señalan algunos desarrollos e implementaciones holónicas; luego se propone una solución para representar requisitos holónicos. En la última sección se presentan las conclusiones del trabajo.

\section{CONCEPTOS DE SISTEMAS HOLÓNICOS}

\section{Sistemas holónicos}

A principios de los años 70 del siglo pasado el filósofo Arthur Koestler propuso el término holón, refiriéndose a una parte y a un todo al mismo tiempo, al analizar el comportamiento de los seres biológicos que pueden actuar de manera autónoma o en sociedad. Un holón se define como un bloque que posee una parte física y una lógica, se puede componer con otros holones y puede pertenecer a un holón más grande [1].

Un holón posee, como propiedades fundamentales, la cooperación y la autonomía. La autonomía del holón es la capacidad de crear y ejecutar sus propios 
planes para conseguir un objetivo y la cooperación se refiere a la sincronización de estrategias de un conjunto de entidades para alcanzar un objetivo común [9]. Según lo anterior, un conjunto de holones forma una holarquía para lograr un objetivo específico. Un Sistema Holónico de Manufactura (HMS, por sus siglas en inglés) es una holarquía que abarca todas las actividades de los procesos de una empresa manufacturera.

Las fábricas de finales de los 80 , que buscaban la optimización de procesos manufactureros, originaron y concibieron los HMS. Desde ese momento se comenzaron a realizar investigaciones hacia la implementación de una holarquía totalmente integrada en las actividades de diseño, producción $\mathrm{y}$ venta de un producto o servicio. Los resultados esperados fueron: toma rápida de decisiones frente a disturbios en el proceso de producción, utilización de algoritmos de optimización para uso eficaz de recursos en todos los niveles, adaptabilidad a cambios en el entorno de la empresa y naturalidad en el flujo de la información, que se expone como una gran ventaja frente a las arquitecturas jerárquicas o heterárquicas de las organizaciones [1].

\section{Arquitecturas de sistemas holónicos}

Son pocas las arquitecturas que los investigadores lograron adoptar para el paradigma holónico. Sin embargo, las dos más representativas son PROSA y ADACOR.

La arquitectura de referencia en HMS es PROSA (Product Resource Order Staff Architecture). PROSA define tres holones básicos enfocados en el conocimiento del producto, las capacidades como recurso y los objetivos que se deben alcanzar. Sus aportes se resumen en la similitud entre los componentes, modelos de descripción de funciones de holones y reducción de complejidad en los procesos manufactureros. Su mayor debilidad es la ausencia de un método orientado hacia la implantación de la arquitectura [2].

En 2005 se propuso ADACOR (ADAptive holonic COntrol aRchitecture). Esta arquitectura realiza aportes en los escenarios industriales, como el manejo de disturbios inesperados en el nivel de planta. ADACOR contiene cuatro holones básicos llamados tarea, recurso, operativo y supervisor, los cuales poseen características de autonomía, reactividad y cooperación [3]. El control de la producción varía entre dos contextos: cuando la planta está en operación normal, el comportamiento es jerárquico y existe una optimización global; cuando existen disturbios, el comportamiento es heterárquico y existe una rápida reacción para no afectar los objetivos asignados. La arquitectura carece de especificaciones para implementar holones en alguna plataforma de programación.

El grupo del Departamento de Mecánica e Ingeniería Industrial de la Universidad de Calgary desarrolla proyectos relacionados con modelos para control inteligente en sistemas de fabricación como MetaMorph I, ABCDE, DIDE y MetaMorph II. Estos proyectos trabajan bajo la arquitectura $\mathrm{HCD}$ (Holonic Control Device) [4]. HCD tiene una arquitectura de cuatro capas: capa deliberativa, capa de funciones de control, capa física y capa de simulación. Su principal aporte es su adaptación a entornos cambiantes con sus dispositivos vistos como holones y la integración física y lógica del holón. Sin embargo, HCD requiere la apropiación de técnicas de desarrollo de software para conformar un proceso metodológico de implementación.

\section{DESARROLLO DE SOFTWARE DE SISTEMAS HOLÓNICOS}

En el desarrollo de software orientado hacia la implementación de sistemas holónicos de producción, son pocas las propuestas que abarcan con precisión el concepto de holón. El principal problema es la carencia de un método estándar de desarrollo, lo que hace que se use un método o una arquitectura en particular. Esto conlleva a algunos analistas usar metodologías con criterios como ciclo de desarrollo por etapas, orientadas a objetos, basadas en agentes, con especificación de requisitos. Sin embargo, son escasos los métodos que presentan validación de requisitos y de las propiedades propias de un holón.

En [10] y [11] se realizaron prototipos de HMS guiados por las arquitecturas, PROSA y Control Metamórfico, respectivamente. Esto significó la ausencia de un desarrollo claro donde se señalaran los pasos a seguir. Además, estos métodos se enfocan inicialmente en una etapa de diseño, es decir, omiten la especificación de requisitos. 
En [1] y [5] se demuestra que las implementaciones de HMS comenzaron orientadas a objetos, con métodos en cascada o iterativo e incremental. El análisis de requisitos se basa en la documentación extraída de la organización interesada, haciendo un mapeo subjetivo entre holones y recursos y construyendo diagramas estructurales y de interacción. La validación se realiza con el interesado, dejando abierta la pregunta de cómo alcanzar más precisión en la identificación de holones y caracterización de sus propiedades.

Actualmente la tendencia de implementación de HMS son los Sistemas Multiagentes (SMA), por las semejanzas entre estos dos paradigmas [6]. Sin embargo, algunas propuestas de agentes poseen debilidades en cuanto a la validación de las características que debe poseer una holarquía de un SMA. En [12] y [13] se menciona la necesidad de validar las propiedades holónicas por medio de simulación. Además, algunas arquitecturas orientadas a agentes presentan debilidades en su estructura jerárquica, cuando un sistema holónico busca un comportamiento jerárquico a nivel global.

En [14] se hace una recopilación de metodologías multiagente como AUML, MaSE, Tropos y KAOS, que contienen fase de análisis. Estos métodos se basan en ingeniería de software orientada a objetos y poseen un modelado semiformal en el análisis, la mayoría de ellos utilizando diagramas de secuencias de UML. En [15] se hace una evaluación comparativa entre los métodos GAIA, Tropos y MaSE, siendo este último el más descriptivo en el desarrollo de software. La evaluación desde el enfoque de modelado arroja como resultado la falta de un vocabulario estable y consistente y la ausencia de la propiedad de escalabilidad en el sistema. Por otro lado, el proceso de desarrollo requiere más cimientos en la verificación y validación del sistema de las distintas etapas.

En [7] se propone un método llamado ANEMONA para desarrollar HMS basado en agentes. ANEMONA es una metodología que, además, incorpora principios de ingeniería de software, presentando un proceso en cada escenario de desarrollo, especialmente en la fase de análisis de requisitos. En esta fase se propone el modelado del agente, de la organización, del entorno, de la interacción, de las metas y tareas y, como componente adicional, la especificación de casos de uso.

El modelo de interacción [16-17] es fundamental para el desarrollo de un sistema holónico en su etapa de análisis de requisitos. Este modelo es importante porque analiza el intercambio de información y las solicitudes entre agentes y/o entre agentes y usuarios humanos. El modelo de interacción tiene varios propósitos: conocer las relaciones sociales de una organización, analizar las dependencias entre los elementos y analizar las metas que se pretende alcanzar a nivel individual o grupal.

La definición de una interacción requiere la identificación de los siguientes elementos [7]:

- Actores en la interacción: Identificación de los participantes iniciadores y colaboradores, determinación de las metas que motivan a cada actor a participar en la interacción.

- Unidades de interacción: Definición y clasificación de los mensajes a utilizar entre los agentes.

- Órdenes de unidades de interacciones: Adaptación de protocolos para la comunicación entre agentes.

- Contexto de la interacción: Objetivo que persigue la interacción y las acciones que realizan los participantes en la interacción.

- Modelo de control: Mecanismos de coordinación.

En la Figura 1 se presenta la simbología del modelo de interacción de ANEMONA.

Entre las limitaciones encontradas en ANEMONA están: carencia en la identificación automática de holones, larga duración en el proceso de construcción de los modelos de análisis y ausencia de mecanismos de validación de estos modelos.

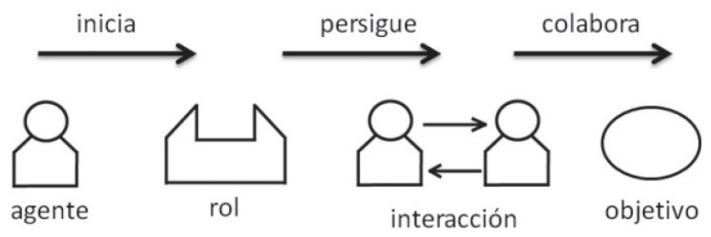

Figura 1. Elementos del modelo de interacción. Adaptación de los autores basada en [7]. 
Este análisis conlleva a concluir que la educción de requisitos para el desarrollo de sistemas holónicos de producción es un proceso largo, con debilidades en la representación e identificación de holones, sin mecanismos de validación de la especificación de requisitos y que no ofrece modelos tratables a nivel computacional. En la Tabla 1 se ofrece un comparativo resumen de criterios usados para el desarrollo de sistemas holónicos según las técnicas, métodos o metodologías más importantes descritas anteriormente.

Tabla 1. Comparativo de criterios de implementación usados en SHM.

\begin{tabular}{|c|c|c|c|c|c|c|c|}
\hline \multirow[b]{2}{*}{ Criterio } & \multicolumn{7}{|c|}{ Técnica, método o metodología } \\
\hline & $\begin{array}{l}\mathbb{1} \\
0 \\
0 \\
0\end{array}$ & 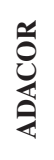 & 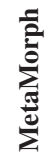 & $\underset{\mho}{\Xi}$ & 苍 & $\frac{\sqrt{1}}{\sum^{\frac{\pi}{2}}}$ & 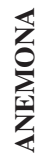 \\
\hline $\begin{array}{l}\text { Desarrollo por } \\
\text { fases }\end{array}$ & No & No & No & Sí & Sí & Sí & Sí \\
\hline $\begin{array}{l}\text { Etapa de } \\
\text { requisitos }\end{array}$ & No & No & No & No & No & No & Sí \\
\hline $\begin{array}{l}\text { Orientada a } \\
\text { objetos }\end{array}$ & No & No & Sí & Sí & Sí & Sí & No \\
\hline $\begin{array}{l}\text { Basada en } \\
\text { agentes }\end{array}$ & No & No & No & Sí & Sí & Sí & Sí \\
\hline $\begin{array}{l}\text { Validación de } \\
\text { requisitos }\end{array}$ & No & No & No & No & No & No & No \\
\hline $\begin{array}{l}\text { Validación } \\
\text { de propiedades } \\
\text { holónicas }\end{array}$ & No & No & No & No & No & No & No \\
\hline $\begin{array}{l}\text { Modelos } \\
\text { tratables } \\
\text { computa- } \\
\text { cionalmente }\end{array}$ & No & No & No & No & No & No & No \\
\hline
\end{tabular}

\section{ESQUEMAS PRECONCEPTUALES (EP)}

\section{Generalidades}

Los esquemas preconceptuales son herramientas útiles para la interpretación del dominio del interesado en un lenguaje controlado, el que posibilita la obtención automática de diagramas UML con el objetivo de elaborar una aplicación de software [8]. Entre las contribuciones encontradas de la ingeniería de requisitos al emplear EP están: identificación de requisitos, simplicidad de la notación de los componentes, facilidad en la comprensión del dominio representado, tratabilidad computacional y validación con el interesado desde etapas tempranas del modelado, el que expone la problemática actual. Estas contribuciones ayudan a construir un software de calidad.

A continuación, en la Figura 2, se describen los componentes básicos que se encuentran en un EP, con el fin de tener claridad sobre la sintaxis empleada. Se debe notar que existe también el concepto de tríada, que es un conjunto de dos conceptos unidos con una relación que puede ser estructural o dinámica.
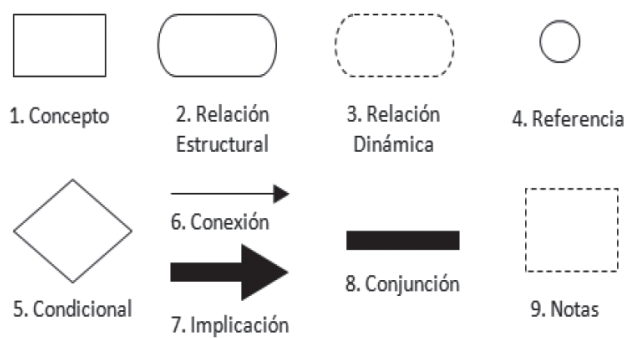

9. Notas

Figura 2. Elementos de un esquema preconceptual. Tomado de [8].

Uno de los beneficios importantes al utilizar los esquemas preconceptuales es que permiten generar un modelo tratable computacionalmente, lo cual se puede reflejar en la obtención automática de nuevos modelos. En [18] se expone la generación automática del diagrama de casos de uso de UML, donde se define un método para la identificación de los elementos de dicho diagrama, tomando como punto de partida los EP. En el mismo sentido, en [19] se presenta una conversión automática de EP en diagramas de secuencia de UML, donde se construyen unas reglas heurísticas de correspondencia entre ambos modelos. Otros diagramas estándar de la ingeniería de software, como los de BPMN (Business Process Modeling Notation), también se pueden generar a partir de los EP.

Las capacidades anteriormente descritas permiten concluir que los EP son apropiados para el análisis de requisitos de una organización, la que busca obtener una buena implementación de software y convertirse en una empresa que cumpla las propiedades de sistemas holónicos. La creación de nuevos modelos a partir de los EP, particularmente, se convierte en una característica altamente deseable para los fines 
que se persiguen en este artículo, si bien se procura la generación automática del modelo de interacción.

\section{REPRESENTACIÓN DE UN PROCESO DE CONFECCIÓN DE PRENDAS DE VESTIR EN ESQUEMAS PRECONCEPTUALES}

En esta Sección se presenta la descripción de un proceso de confección de prendas de vestir y su ilustración en EP (véase la Figura 3). El objetivo es conocer la potencia de esta representación gráfica que es simple y entendible para máquinas y humanos.

En una empresa textil existen cinco trabajadores que realizan distintas actividades para comercializar, en última instancia, prendas de vestir, que en este caso se denominarán productos. La producción se caracteriza por tener una cantidad de productos y unas fechas de inicio y entrega. En el nivel más bajo de la escala empresarial se encuentran los siguientes actores, quienes realizan el producto físico como tal: (a) el empleado de corte, quien inicia la confección de la prenda en su forma más básica, tarea que implica esperar la entrega que hace el encargado del almacén de la materia prima. (b) El empleado de costura y acabados, quien se encarga de pedir las prendas básicas al empleado de corte para terminar de fabricar la prenda y, finalmente, entregarla al jefe de producción. (c)

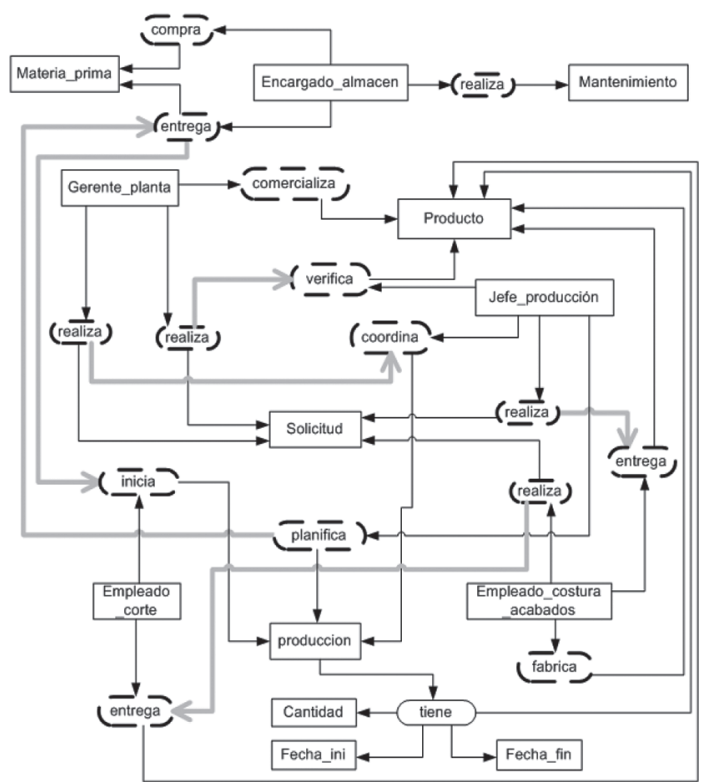

Figura 3. Proceso de confección expresado en EP. Elaboración propia de los autores.
El encargado de almacén, quien se encarga de tres actividades: la compra y entrega de materia prima y la ejecución del mantenimiento del almacén. Por otro lado, en un nivel más alto, se encuentran el gerente de planta y el jefe de producción. El primero se encarga de comercializar los productos terminados de la empresa y exigir al segundo que efectúe las actividades de verificación del producto y planificación de la producción. Esta descripción en particular muestra la manera en que se encadenan las actividades que involucra varios actores, usando el elemento implicación de los EP.

\section{REGLAS DE TRANSFORMACIÓN PARA GENERAR UN MODELO DE INTERACCIÓN A PARTIR DE EP}

En este trabajo se proponen cinco reglas de transformación para la generación de un modelo de interacción a partir de EP. Estas reglas buscan eliminar la subjetividad que puede tener un analista al enfrentarse con diferentes niveles de complejidad en diferentes procesos organizacionales. Asimismo, es útil para los analistas observar que tan factible es para un actor convertirse en holón, validando las interacciones que este tiene como autonomía, cooperación y reactividad. Se debe aclarar que estas reglas son heurísticas.

\section{Regla 1: Interacción simple}

Cuando existe una relación dinámica con una implicación hacia otra relación dinámica que efectúa un actor diferente al de la tríada anterior (Figura 3) se puede conformar una interacción simple de la forma: A1 inicia colaboración con A2 efectuando D2 C2 (véanse las Figuras 4 y 5).

\section{Regla 2: Interacción múltiple}

Al disponer de varias tríadas dinámicas con implicaciones entre ellas y siendo el concepto origen siempre distinto (Figura 5), se puede deducir una cadena de interacciones simples denotada de la siguiente forma: A1 inicia colaboración con A2 efectuando D2 C2, y A2 inicia colaboración con A3 efectuando D3 C3 (Figura 6). Cabe mencionar que es válido que $\mathrm{C} 1$ puede ser igual a $\mathrm{C} 3$.

\section{Regla 3: Interacción con múltiple cooperación} Cuando existe una tríada dinámica y esta implica dos o más acciones dinámicas que realizan otros actores (Figura 7), entonces se concluye que existe 


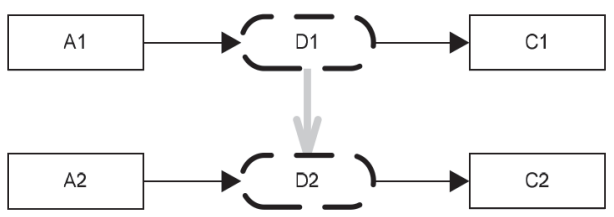

Figura 4. Regla 1 en EP. Elaboración propia de los autores.

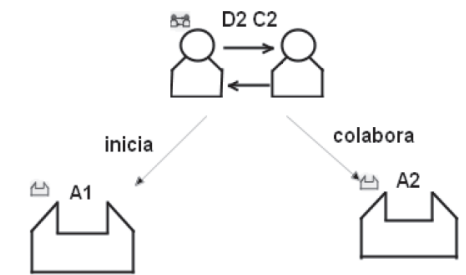

Figura 5. Regla 1 en modelo de interacción. Elaboración propia de los autores.

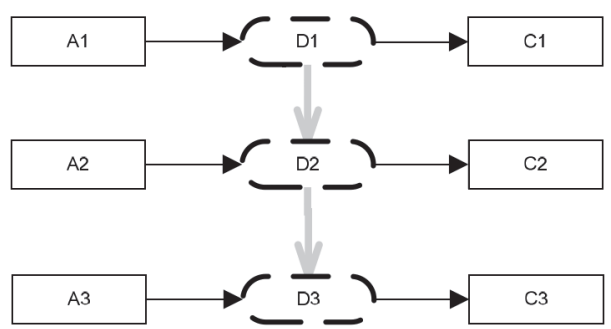

Figura 6. Regla 2 en EP. Elaboración propia de los autores.
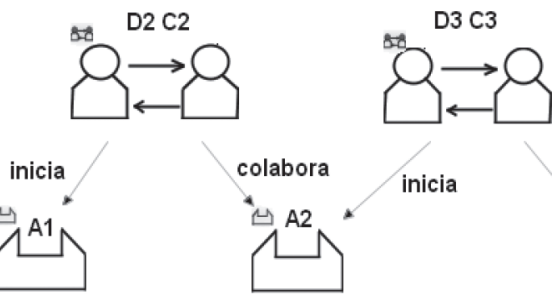
inicia

colabora

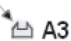

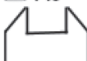

Figura 7. Regla 2 en modelo de interacción. Elaboración propia de los autores.

un actor que inicia dos o más cooperaciones con otros actores, lo que se denotaría como: A2 inicia colaboración con A1 efectuando D1 C1, y A2 inicia colaboración con A3 efectuando D3 C3 (Figura 8).

\section{Regla 4: Interacción con una cooperación}

Cuando existe una tríada dinámica y esta implica dos o más acciones dinámicas que realizan otros actores, con la particularidad que sus acciones son iguales y apuntan al mismo concepto destino (Figura 9), entonces se concluye que existe un actor iniciador a una cooperación con varios actores, lo

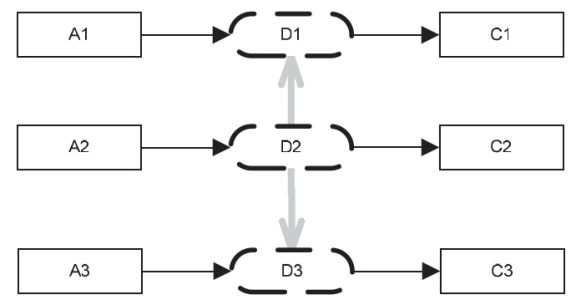

Figura 8. Regla 3 en EP. Elaboración propia de los autores.

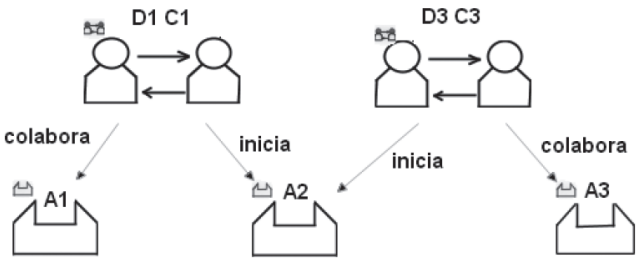

Figura 9. Regla 3 en modelo de interacción. Elaboración propia de los autores.

que se denotaría como: A2 inicia colaboración con A1 y A3 efectuando D1 C1 (Figura 10).

\section{Regla 5: Interacción con requisitos}

Si se presenta una relación de logro acoplada con una tríada dinámica (Figura 11), se puede deducir que un rol o agente persigue un requisito en el diagrama de interacción, lo que se puede denotar así: A1 persigue O1 D1 C1 (Figuras 12 y 13).

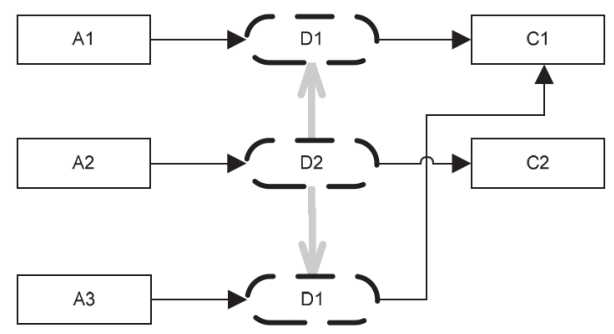

Figura 10. Regla 4 en EP. Elaboración propia de los autores.
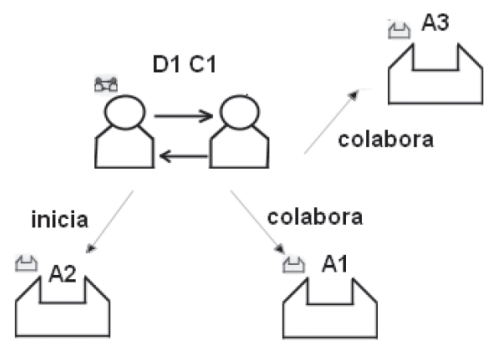

Figura 11.Regla 4 en modelo de interacción. Elaboración propia de los autores. 


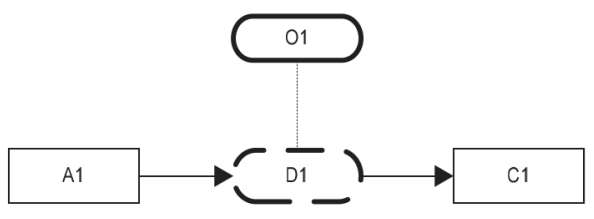

Figura 12. Regla 5 en EP. Elaboración propia de los autores.

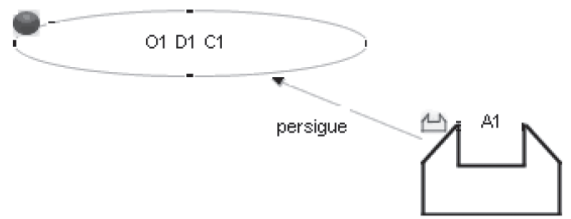

Figura 13. Regla 5 en modelo de interacción. Elaboración propia de los autores.

\section{CASO DE ESTUDIO}

El siguiente caso de estudio ilustra la manera de aplicar determinadas reglas al EP de la Figura 3 para hallar, finalmente, un diagrama de interacción útil en el proceso de desarrollo de un sistema holónico, específicamente para identificar las relaciones sociales en una organización.

El proceso de confección de prendas de vestir presenta algunas dependencias de actividades entre los empleados de la empresa. Analizando el EP se observa que es factible aplicar la regla 1 varias veces y la regla 2 una sola vez. En el primer caso se analiza la siguiente frase expresada en el EP: "Gerente_planta realiza solicitud, entonces Jefe_producción coordina producción". En el diagrama de interacción, por su parte, tal frase se refleja en la interacción "coordina producción", la que la inicia, realmente, el gerente de planta, mientras que la ejecuta (colabora) el jefe de producción. Este análisis se hace de manera similar para las relaciones del EP que apliquen a la regla de interacción simple. En el segundo caso la frase en EP "Jefe_producción planifica producción, entonces Encargado_almacén entrega Materia_prima, entonces Empleado_corte inicia producción", se observa que se ajusta a la precondición de la regla 2 , interacción múltiple. En el nuevo modelo se presenta la siguiente secuencia de interacciones: "Jefe_producción inicia la entrega de Materia_ prima, que ejecuta el Encargado_almacén quien, a su vez, inicia la Producción con la colaboración de Empleado_corte".
El diagrama de interacción de la Figura 14 presenta las relaciones de dependencia del personal de la compañía textil. Este diagrama es importante en la fase de diseño para conocer los posibles protocolos y mensajes de comunicación entre los actores aquí representados. La transformación de los modelos permite afirmar que existen cinco roles en la empresa textil. Todos ellos, excepto Empleado_corte, son posiblemente holones, debido a que todos ellos inician una interacción en particular y todos ellos colaboran entre sí. Es en este momento cuando el analista examina otros factores de análisis como otro tipo de información de la organización y otros diagramas de análisis. Se busca que el analista la decisión de si un rol finalmente cumple con las propiedades de un holón o no. Sin embargo, el diagrama preliminar que se obtuvo es de gran ayuda para tomar dicha decisión.

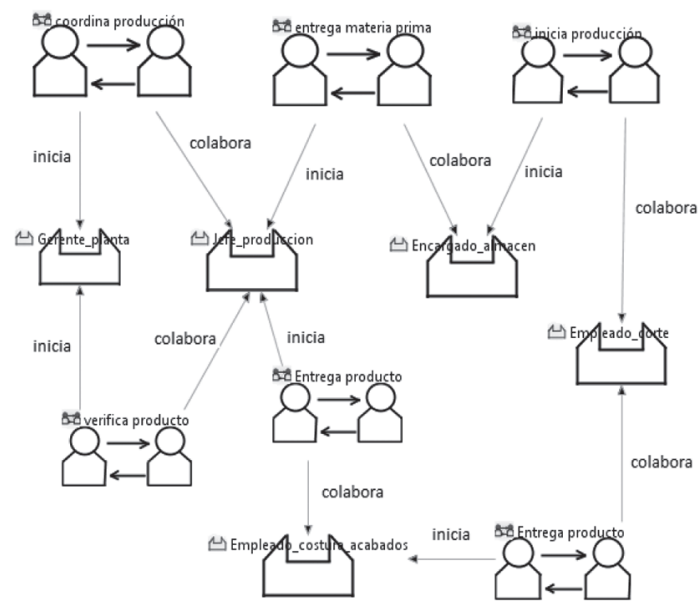

Figura 14. Diagrama de interacción del proceso de confección expresado en Figura 3. Elaboración propia de los autores.

\section{CONCLUSIONES}

Existen algunas limitaciones identificadas en la fase de análisis de requisitos de las metodologías holónicas, como ausencia de validación, largo tiempo en la construcción de modelos de análisis holónicos, carencia de métodos que permitan identificar rápidamente holones en un proceso organizacional y problemas de subjetividad en el análisis del mismo. Debido a esto se propuso en este artículo la representación de los requisitos de los sistemas holónicos mediante esquemas preconceptuales para eliminar los problemas de ambigüedad en 
las descripciones de un proceso organizacional. Se eligieron los esquemas preconceptuales para la representación, debido a que implícitamente contienen una gran cantidad de diagramas en su interior que se pueden generar automáticamente, incluyendo los diagramas correspondientes a la notación BPMN, considerada un estándar en la actualidad. Además, los esquemas preconceptuales posibilitan la tratabilidad computacional y poseen un lenguaje cercano al natural, que suelen emplear los analistas para lograr la validación con los interesados.

También se formularon cinco reglas que transforman un EP en un modelo de interacción de sistemas holónicos. Esta propuesta automatiza la identificación de posibles holones en un proceso empresarial, elimina problemas de subjetividad al analizar procesos complejos, permite comprender las interacciones de todos los actores dentro del proceso (véanse las Reglas 1 a 4) en busca de propiedades holónicas como autonomía, reactividad y cooperación y finalmente, proporciona información de qué requisitos debe perseguir un rol (véase la Regla 5). Así, los analistas de sistemas holónicos pueden reducir el tiempo de análisis de un proceso a partir del discurso de un interesado. El caso de estudio del proceso de confección demostró cómo aplicar las reglas a los elementos de un EP para obtener información útil en un escenario holónico.

Como trabajo futuro se plantea la construcción de nuevas reglas de transformación y su adecuada implementación a nivel de software para generar, automáticamente y de manera gráfica, el modelo de interacción a partir de un esquema preconceptual. También se pueden generar otros diagramas pertenecientes a diversas metodologías para el diseño de sistemas holónicos.

\section{REFERENCIAS}

[1] R. Babiceanu and F. Chen. "Development and applications of holonic manufacturing systems: a survey". Journal of Intelligent Manufacturing. Vol. 17, Issue 1, pp. 111131. February, 2006. ISSN: 1572-8145. DOI: $10.1007 / \mathrm{s} 10845-005-5516-\mathrm{y}$.

[2] H. Van Brussel, J. Wyns, P. Valckenaers, L. Bongaerts and P. Peeters. "Reference architecture for holonic manufacturing systems: PROSA". Computers in industry.
Vol. 37, Issue 3, pp. 255-274. November, 1998. ISSN: 0166-3615. DOI: 10.1016/ S0166-3615(98)00102-X.

[3] P. Leitao and F. Restivo. "ADACOR: A holonic architecture for agile and adaptive manufacturing control". Computers in industry. Vol. 57, Issue 2, pp. 121-130. February, 2006. ISSN: 0166-3615. DOI: 10.1016/j.compind.2005.05.005.

[4] R.W. Brennan, K. Hall, V. Marik, F. Maturana and D.H. Norrie. "A Real-Time Interface for Holonic Control Devices". Holonic and Multi-Agent Systems for Manufacturing, eds. V. Marík, D. McFarlane and P. Valckenaers. Springer Berlin. Heidelberg, Germany. Vol. 2744, pp. 10881088. September, 2003. ISSN: 0302-974. DOI: 10.1007/978-3-540-45185-3_3.

[5] L. Rannanjärvi and T. Heikkilä. "Software development for holonic manufacturing systems". Computers in industry. Vol. 37, Issue 3, pp. 233-253. November, 1998. ISSN: 0166-3615. DOI: 10.1016/ S0166-3615(98)00101-8.

[6] A. Giret, V. Julián and V. Botti. "Aplicaciones Industriales de los Sistemas Multiagente". Agentes Software y Sistemas Multi-Agente. Conceptos, Arquitecturas y Aplicaciones, eds. Prentice-Hall. Vol. 1, pp. 186-203. Madrid, España. 2005. ISBN: 84-205-4367-5.

[7] A. Giret and V. Botti. "Engineering Holonic Manufacturing Systems". Computers in industry. Vol. 60, Issue 6, pp. 428-440. August 2009. ISSN: 0166-3615. DOI: 10.1016/j. compind.2009.02.007.

[8] C. Zapata. "Definición de un esquema preconceptual para la obtención automática de esquemas conceptuales de UML". Tesis doctoral. Universidad Nacional de Colombia. Medellín, Antioquia, Colombia. 2007.

[9] F.-T. Cheng, C.-F. Chang and S.-L. Wu. "Development of holonic manufacturing execution systems". Journal of Intelligent Manufacturing. Vol. 15, Issue 2, pp. 253267. April, 2004. ISSN: 1572-8145. DOI: 10.1023/B:JIMS.0000018037.63935.a1.

[10] J. Zimmermann and L. Mönch. "Design and Implementation of Adaptive Agents for Complex Manufacturing Systems". Holonic and Multi-Agent Systems for Manufacturing, eds. V. Marík, V. Vyatkin 
and A. Colombo. Springer Berlin. Heidelberg, Germany. Vol. 4659, pp. 269280. September, 2007. ISSN: 0302-9743. DOI: 10.1007/978-3-540-74481-8_26.

[11] S. Balasubramanian, R.W. Brennan and D.H. Norrie. "Requirements for holonic manufacturing systems control". In Proceedings of 11th International Workshop on Database and Expert Systems Applications, pp. 214-218. London, United Kingdom. ISBN: 0-7695-0680-1.

[12] L. Monostori and B. Kadar. "Agent-based control of manufacturing systems". In Proceedings of the Second International Conference on Intelligent Processing and Manufacturing of Materials, IPMM '99, pp. 131-137. Honolulu, Hawaiian. ISBN: 0-7803-5489-3.

[13] F.P. Maturana, R.J. Staron, P. Tichý, P. Šlechta and P. Vrba. "A Strategy to Implement and Validate Industrial Applications of Holonic Systems". Holonic and Multi-Agent Systems for Manufacturing, eds. V. Marík, R.W. Brennan and M. Pechoucek. Springer Berlin. Heidelberg, Germany. Vol. 3593, pp. 111120. August, 2005. ISSN: 0302-9743. DOI: 10.1007/11537847_10.

[14] H. Xu and Z. Jin. "An Agent-Oriented Requirement Graphic Symbol Representation and Formalization Modeling Method". In
WRI World Congress on Computer Science and Information Engineering, pp. 569574. Los Angeles, USA. 2009. ISBN: 978-0-7695-3507-4.

[15] A. Sturm and O. Shehory. "A Comparative Evaluation of Agent-Oriented Methodologies". Methodologies and Software Engineering for Agent Systems, eds. F. Bergenti, M.-P. Gleizes and F. Zambonelli. Springer US. Vol. 11, pp. 127-149. 2004. ISSN: 15682617. DOI: 10.1007/1-4020-8058-1_9.

[16] A. Giret and V. Botti. "Analysis and design of holonic manufacturing systems". In Proceedings of the 18th International Conference on Production Research, pp. 1-10. University of Salerno. Italy. 2005.

[17] A. Giret and V. Botti. "From system requirements to holonic manufacturing system analysis". International journal of production research. Vol. 44, Issue 18, pp. 3917-3928. September, 2006. ISSN: 0020-7543. DOI: 10.1080/00207540600696336.

[18] C. Zapata, P. Tamayo and F. Arango. "Conversión de esquemas preconceptuales a diagrama de casos de uso empleando AToM3”. Dyna. Vol. 153, Issue 2, pp. $237-$ 251. 2007. ISSN: 0012-7353.

[19] C. Zapata and G. Garcés. "Generación del diagrama de secuencias de UML 2.1. desde esquemas preconceptuales". Revista EIA. $\mathrm{N}^{\mathrm{o}} 10$, pp. 89. 2008. ISSN: 1794-1237. 